\title{
ATP-Binding Cassette (ABC) Transporter Genes in Plant-Parasitic Nematodes: An Opinion for Development of Novel Control Strategy
}

\author{
Rinu Kooliyottil ${ }^{1,2 *}$, Koushik Rao Gadhachanda ${ }^{3}$, Nejra Solo $^{2}$ and \\ Louise-Marie Dandurand ${ }^{2 *}$
}

${ }^{1}$ Citrus Budwood Registration Program, Division of Plant Industry, Florida Department of Agriculture and Consumer Services, La Crosse, FL, United States, ${ }^{2}$ Department of Entomology, Plant Pathology and Nematology, University of Idaho, Moscow, ID, United States, ${ }^{3}$ Independent Researcher, Cheshire, CT, United States

Keywords: plant parasitic nematodes, resistance, xenobiotic metabolism, $A B C$ transporters, gene silencing

\section{OPEN ACCESS}

Edited by:

Holger Heuer

Julius Kühn-Institut, Germany

Reviewed by:

Krzysztof Wieczorek

University of Natural Resources and

Life Sciences Vienna, Austria

André Bertran,

Wageningen University and

Research, Netherlands

*Correspondence:

Rinu Kooliyotti

rinu.kooliyottil@fdacs.gov:

rinukmicro@gmail.com

Louise-Marie Dandurand

Imd@uidaho.edu

Specialty section:

This article was submitted to

Plant Pathogen Interactions,

a section of the journal

Frontiers in Plant Science

Received: 11 July 2020

Accepted: 05 October 2020

Published: 20 November 2020

Citation:

Kooliyottil R, Rao Gadhachanda K, Solo N and Dandurand L-M (2020)

ATP-Binding Cassette (ABC)

Transporter Genes in Plant-Parasitic

Nematodes: An Opinion for

Development of Novel Control

Strategy. Front. Plant Sci. 11:582424.

doi: 10.3389/fp/s.2020.582424

\section{INTRODUCTION}

Nematodes and plants have interacted for millions of years. Over the years, plant-parasitic nematodes (PPNs) have developed sophisticated mechanisms to overcome the immune response from plants, being able to establish successful parasitism in susceptible host plants. Today, nematodes have complex feeding structures along with other highly adaptive features, which suit their environment (Ali et al., 2017). Along with nematode evolution, plants have also adapted to recognize changes in pathogens for continued effective defense response. Initial contact with the PPNs triggers immune response in the host plant system which includes the release of toxic molecules. To put a bridle on this immune response, PPNs trigger pivotal cytoprotective mechanisms, such as antioxidant and detoxification pathways (Gillet et al., 2017). Mechanisms of these pathways have been studied in PPNs, and the specific genes involved have been targeted for gene silencing research in view of developing novel control measures (Gillet et al., 2017; Qiu et al., 2019). However, one of the important group of proteins involved in detoxification pathways known as ATP-binding cassette (ABC) transporters have not been studied until recently in PPNs. This opinion article focuses on the current knowledge and prospects of $\mathrm{ABC}$ transporters in PPNs.

\section{PLANT-NEMATODE INTERACTIONS}

Plants use a set of induced and constitutive strategies to protect themselves against pathogens. The protective measures are activated when pathogen-derived compounds called pathogen-associated molecular pattern (PAMPs) are recognized. In resistant plants, PAMP perception activates pattern-triggered immunity (PTI), which initiates signals that facilitate resistance to the growth of pathogens (Jones and Dangl, 2006). In plantparasitic nematodes, small molecules called ascarosides, an evolutionarily conserved family of nematode pheromones, are known to induce microbe-associated molecular pattern (MAMP)-triggered immunity (Manosalva et al., 2015). An important ascaroside in plantparasitic nematodes, ascr\#18, was reported earlier to induce defense signaling pathways in Arabidopsis, tomato, potato, and barley to viral, bacterial, oomycete, fungal, and nematode infections (Manosalva et al., 2015). In resistant plants, the nucleotide-binding site-leucine-rich repeat (NB-LRR) proteins recognize the pathogen effectors which leads to effector-triggered immunity (ETI). Effector-triggered immunity directs one of the most effective plant 
defense mechanisms called the hypersensitive response (HR) (Bigeard et al., 2015), whereby a few cells surrounding the ingressing pathogen or pest die to ward off the pathogen. Potato cyst nematode Globodera pallida secretes protein RBP-1, which is known to induce defense responses, including cell death typical of HR through the NB-LRR protein Gpa2 (Sacco et al., 2009). Some early signs of HR are rapid influxes of free calcium $\left(\mathrm{Ca}^{2+}\right)$, production of the reactive oxygen species (ROS), nitric oxide, and changes in the phytohormone production (Garcia-Brugger et al., 2006; Lozano and Smant, 2011). Among several important roles during the plant defense response, rapid influxes of $\mathrm{Ca}^{2+}$ are considered to be crucial for the activation of the NADPH oxidase found on the membrane of the plant cell (Kadota et al., 2015). Oxidases produce extracellular ROS, which initiate a cascade of events leading to an oxidative burst (Lozano and Smant, 2011). An oxidative burst along with the production of ROS is also an important part of the plant defense, since ROS create a cytotoxic environment for the pathogen or pest and also act as signaling molecules for local and systemic defense responses (Rosso, 2009; Gillet et al., 2017).

\section{XENOBIOTIC METABOLISM TO COUNTERACT PLANT RESISTANCE}

Pathogens and pests, including plant-parasitic nematodes, use antioxidant, and detoxification pathways in order to protect themselves and establish successful infections. Pathogens with endoparasitic lifestyles, which are exposed to the plant defense response for a considerable amount of time during their life cycle, use this mechanism very efficiently to overcome the host resistance (Robertson et al., 2000). The response of nematodes to the oxidative stress is predominantly regulated by transcription factors, daf-16 and $s k n-1$, which are also important for the survival of nematodes in their pre-parasitic stage (Gillet et al., 2017). These transcription factors are responsible to activate various antioxidant and detoxifying pathways, which function to avoid formation of the highly toxic ROS, to control and neutralize levels of ROS, as well as to prevent cellular damage due to oxidative stress (Callahan et al., 1988; Rosso, 2009; Gillet et al., 2017). In the nematode antioxidant pathway, daf16 and $s k n-1$ act together to regulate the expression of genes such as superoxide dismutase (SOD) and catalase (CTL), genes that encode glutathione peroxidases (GPX) and peroxiredoxin (PRDX) (Gillet et al., 2017). Xenobiotic/endobiotic detoxification pathway, also mediated by daf-16 and/or skn-1, is activated against internal or external toxic compounds (Gillet et al., 2017) and has a role in the detoxification and excretion of these compounds (Lindblom and Dodd, 2009). Basso et al., 2020 used in planta RNAi technology to silence the daf-16 and skn-1 transcription factors and achieved significant resistance to root knot nematode Meloidogyne incognita. Silencing of daf-16 and skn-1 also resulted in the downregulation of important genes involved in detoxification pathway of the nematode.

There are three phases of xenobiotic metabolism. Phase I, which mainly involves cytochrome P450s, makes xenobiotics and endobiotics more soluble, while phase II is a detoxification step. In this phase, enzymes such as uridine dinucleotide phosphate glucuronosyltransferases (UGT) and glutathione S-transferases (GST) catalyze conjugate formation of xenobiotics and endobiotics with glutathione, amino acids, acetate, sulfate, propionate, or phosphate marking them for excretion (Kurutas, 2015; Laing et al., 2015). Most commonly this involves conjugation to glutathione (GSH), which is a tripeptide ( $\gamma$-GluCys-Gly) that has a major role in the processes of detoxification and redox buffering. In its reduced form, it acts as a nucleophile that attacks electrophilic carbon, nitrogen, or sulfur atom on the toxic non-polar compound (Edwards et al., 2000; Islam et al., 2017). Together with other antioxidants, such as ascorbate, $\alpha$-tocopherol, and cysteine, it is an important aspect of nonenzymatic protection against oxidative stress (Kurutas, 2015). In animals, including nematodes, phase III involves excretion of these conjugates by $\mathrm{ABC}$ transporters, which do not belong to the family of detoxifying enzymes (Lindblom and Dodd, 2009). ABC transporters play a major role in the pumping of xenobiotic and endogenous metabolites through extra- and intracellular membranes, which helps to reduce the cellular concentrations of toxic compounds. Recent developments in xenobiotic metabolism in plant-parasitic nematodes give evidence of a multi-layered strategy using various effectors in a systematic way to protect from host-derived xenobiotic compounds (Espada et al., 2016; Lilley et al., 2018).

\section{ABC TRANSPORTERS IN PLANT-PARASITIC NEMATODES}

A strongly conserved ATP-binding motif and highly conserved functional arrangement in membranes are the signature keys of the $\mathrm{ABC}$ transporters present in different organisms, from bacteria to humans (Higgins, 1992; Childs and Ling, 1994; Linton and Higgins, 1998). With 60 genes, ABC transporters constitute the largest family of transporters in the genome of Caenorhabditis elegans, where they have been shown to be associated with drug resistance (Sheps et al., 2004; Pohl et al., 2011; Ardelli, 2013). Furthermore, elevated expression of $\mathrm{ABC}$ transporter genes has been reported in animal-parasitic nematodes (APNs) in association with resistance to drugs such as ivermectin and macrocyclic lactones (Xu et al., 1998; Prichard and Roulet, 2007; Stitt et al., 2011). However, very few reports on the role of $\mathrm{ABC}$ transporters in PPNs exist. The genome of the PPN Bursaphelenchus xylophilus has 106 $\mathrm{ABC}$ transporters, which is almost double the number found in the genome of C. elegans and about three times more than what is found in the genome of M. incognita (Kikuchi et al., 2011). Upregulation of Bursaphelenchus ABC transporter genes in response to a $\alpha$-pinene, a monoterpene produced by plants in response to pathogen attack, has been found recently (Li et al., 2019). Diao et al., 2020 investigated the multi-drug-resistant protein coding (MDR) genes in B. xylophilus with a focus on screening nematicides (emamectin benzoate, avermectin, and matrine) for the control of these devastating nematodes and found that members of the MDR gene family encode the $A B C$ transporter and the $\mathrm{ABC}$ transporter transmembrane region. 


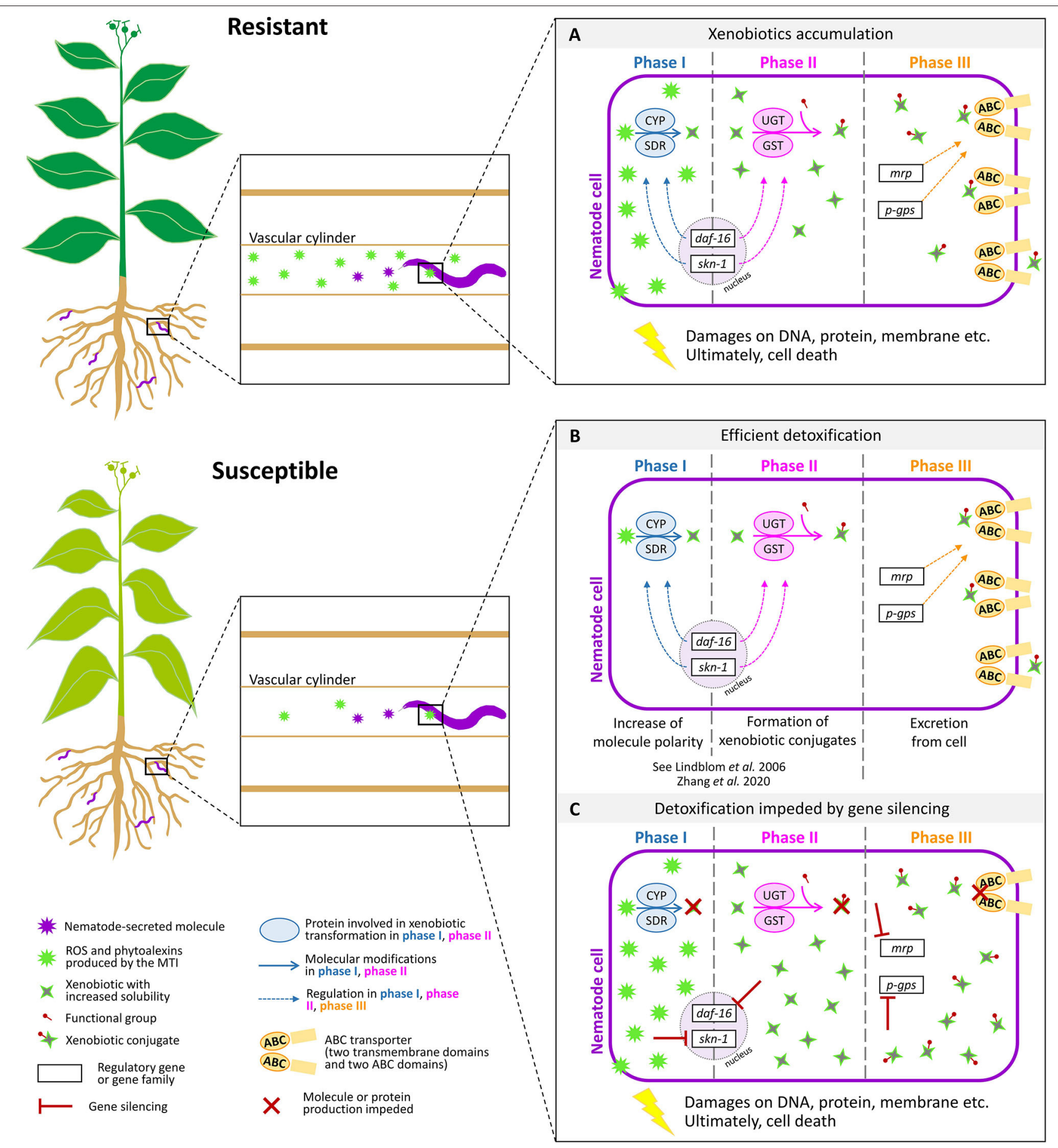

FIGURE 1 | Response of plant-parasitic nematodes in resistant and susceptible plants. Resistant plants produce reactive oxygen species (ROS) and phytoalexins through the microbe- or pathogen-associated molecular patterns (MAMPs or PAMPs), in response to the detection of nematode-secreted molecules. Susceptible plants produce less compounds to defend the nematode presence. Plant-parasitic nematodes have evolved xenobiotic metabolic pathways to counteract the cytotoxic defense response of plants (A). During phase I of xenobiotic metabolism, the polarity and solubility of xenobiotics is increased, often by oxidation, reduction, or hydrolysis reactions. In phase II, a functional group is added to form xenobiotic conjugates. The production of proteins involved in phase I and II is orchestrated by two transcription factors, daf-16 and skn-1. In phase III, ABC transporters excrete xenobiotic conjugates from the cell. The expression of genes coding for ABC transporters is regulated by gene families such as MRP and P-GPS (B). Silencing genes involved in xenobiotic detoxification compromise nematode cell survival. The model proposes that silencing genes involved in phase III would lead to the impediment of the production of ABC transporters and thus to a lethal accumulation of xenobiotic conjugates that could not be excreted from the cell (C). ABC, ATP-binding cassette; CYP, cytochrome P450; GST, glutathione S-transferase; SDR, short-chain dehydrogenase; UGT, UDP-glucuronosyl or UDP-glycosyltransferase; daf-16, dauer formation 16; skn-1, skinhead transcription factor-1; mrp, multi-drug resistance protein; MTI, MAMP-triggered immunity; p-gps, P-glycoproteins. Credits: root shape was derived from Figure 1 in Schouteden et al. (2015). 
Fu et al. (2020) recently compared gene expression patterns between hydrated and 24-h desiccated nematodes of the foliar nematode Aphelenchoides fragariae. This study shows differential expression of detoxification genes, including the $p g p$-14-like multi-drug resistance protein (MRP/PGP), which is part of the $\mathrm{ABC}$ transporter system (Figure 1).

Interactions of $G$. pallida with its natural host Solanum tuberosum and a resistant plant Solanum sisymbriifolium was investigated previously (Kooliyottil et al., 2016, 2019). A hypersensitive response (HR) was evident as early as $24 \mathrm{~h}$ post infestation in the root cells of S. sisymbriifolium (Kooliyottil et al., 2016). Transcriptome analysis of G. pallida juveniles isolated from resistant $S$. sisymbriifolium or the susceptible host, potato, $24 \mathrm{~h}$ post infestation showed expression of several genes related to the xenobiotic metabolism (Kooliyottil et al., 2019). G. pallida isolated from S. tuberosum and S. sisymbriifolium showed that there were 18 G. pallida ABC transporters (GPLIN_000375400, GPLIN_001624000, GPLIN_000079800, GPLIN_000593000, GPLIN_000607700, GPLIN_000662100, GPLIN_000762600, GPLIN_000764700, GPLIN_000165600, GPLIN_001513300, GPLIN_000934100, GPLIN_001038000, GPLIN_001558100, GPLIN_001072100, GPLIN_000043700, GPLIN_001213800, GPLIN_001600700, and GPLIN_000055000) expressed during plant infection (Kooliyottil et al., 2019). Although not significantly different, six $\mathrm{ABC}$ transporter genes were over-expressed when G. pallida infected resistant plant $S$. sisymbriifolium, and four were over-expressed when infecting susceptible $S$. tuberosum, and the rest were expressed with similar values when infecting both plant species (Kooliyottil et al., 2019).

\section{PROSPECTS: NEMATODE ABC TRANSPORTERS AS POTENTIAL TARGET TO CONTROL PLANT-PARASITIC NEMATODES}

Transcriptome information of PPNs isolated from resistant plant species is scanty. Most of the available PPN transcriptome information comes from studies on susceptible plant species. Data obtained from nematode-infected resistant plants is scanty and does not provide evidence on expression of $\mathrm{ABC}$ transporters (Shukla et al., 2018; Cotton et al., 2014). Although our results (Kooliyottil et al., 2019) are not showing statistically significant difference in G. pallida ABC transporter expression when infecting a resistant or susceptible plant species, the expression of genes coding for $\mathrm{ABC}$ transporter proteins suggests that they play a role in plant infection. Genes associated with xenobiotic biodegradation pathways were upregulated when G. pallida

\section{REFERENCES}

Ali, M. A., Azeem, F., Li, H., and Bohlmann, H. (2017). Smart parasitic nematodes use multifaceted strategies to parasitize plants. Front. Plant Sci. 8:1699. doi: 10.3389/fpls.2017. infected resistant S. sisymbriifolium. This attribute toward the nematode's defense response to secondary metabolites is induced by S. sisymbriifolium upon invasion (Kooliyottil et al., 2019). This is evident from our previous study that the juveniles were unable to detoxify the secondary metabolites, leading to death within the root during the early stages of parasitism (Kooliyottil et al., 2016). The hostile environment in a resistant plant cell could be compared to the chemicals/drugs used for treatment against APNs. As a response to drugs, over-expression of $\mathrm{ABC}$ transporters was reported in several nematodes including $C$. elegans (Ardelli, 2013). Furthermore, when ABC transporter genes $m r p-1$ and $p g p-1$ were silenced in C. elegans, the sensitivity toward the drugs or heavy metal ions was increased as compared to the wild type (Broeks et al., 1996). Increased expression of ABC transporter system genes $M R P$ and $P G P$ in C. elegans resistant to ivermectin was reported by James and Davey (2009).

Several reports are available on the xenobiotic metabolism in PPNs, and these genes have been successfully used for gene silencing research with a focus on developing PPN-resistant crop varieties. An investigation about $\mathrm{ABC}$ transporters in PPNs, especially when interacting with resistant plant species, may provide useful information about how nematodes are able to overcome plant defenses (Figure 1). Considering the existing knowledge on the importance of $\mathrm{ABC}$ transporters in APNs, characterization of $\mathrm{ABC}$ transporter genes may contribute to the identification of gene targets for silencing and may provide novel strategies for PPN control. Silencing ABC transporters in APNs has proven effective and is considered as a great tool to control these parasites. We propose further research to determine the role of $\mathrm{ABC}$ transporters and other genes involved in xenobiotic response of PPNs to stress conditions as may be encountered in resistant plants. Understanding the role and mechanisms of ABC transporters in PPNs will be helpful to identify the strategies for achieving sustainable pest control and may even facilitate development of PPN-resistant plants.

\section{AUTHOR CONTRIBUTIONS}

RK developed the concept. RK and KR analyzed the data. RK, $\mathrm{KR}$, NS, and L-MD contributed to discussions and writing the manuscript. All authors contributed to the article and approved the submitted version.

\section{ACKNOWLEDGMENTS}

The support received from Dr. Morgane B. Gillard for the illustration of xenobiotic metabolism in plant-parasitic nematodes in resistant and susceptible plants is acknowledged. 
the control and management of Meloidogyne incognita. Sci. Rep. 1:6991. doi: $10.1038 /$ s41598-020-63968-8

Bigeard, J., Colcombet, J., and Hirt, H. (2015). Signaling mechanisms in pattern-triggered immunity (PTI). Mol. Plant 8, 521-539. doi: 10.1016/j.molp.2014.12.022

Broeks, A., Gerrard, B., Allikmets, R., Dean, M., and Plasterak, R. H. (1996). Homologues of the human multidrug resistance genes MRP and MDR contribute to heavy metal resistance in the soil nematode Caenorhabditis elegans. EMBO J. 15, 6132-6143. doi: 10.1002/j.1460-2075.1996.tb0 1001.x

Callahan, H. L., Crouch, R. K., and James, E. R. (1988). Helminth anti-oxidant enzymes: a protective mechanism against host oxidants?. Parasitol. Today 4, 218-225. doi: 10.1016/0169-4758(88) 90162-7

Childs, S., and Ling, V. (1994). "The MDR superfamily of genes and its biological implications," in Important Advances in Oncology. eds V. T. DeVita, S. Hellman, and S. A. J. B. Rosenberg (Lippincott: Philadelphia), 21-36.

Cotton, J. A., Lilley, C. J., Jones, L. M., Kikuchi, T., Reid, A. J., Thorpe, P., et al. (2014). The genome and life-stage specific transcriptomes of Globodera pallida elucidate key aspects of plant parasitism by a cyst nematode. Genome Biol. 15:R43. doi: 10.1186/gb-2014-15$3-\mathrm{r} 43$

Diao, J., Hao, X., and Ma, W. (2020). Bioinformatics analysis of structure and function in the MRP gene family and its expression in response to various drugs in Bursaphelenchus xylophilus. J. For. Res. doi: 10.1007/s11676-01901086-6

Edwards, R., Dixon, D. P., and Walbot, V. (2000). Plant glutathione S-transferases: enzymes with multiple functions in sickness and in health. Trends Plant Sci. 5, 193-198. doi: 10.1016/S1360-1385(00)01601-0

Espada, M., Silva, A. C., Eves van den Akker, S., Cock, P. J. A., Mota, M., and Jones, J. T. (2016). Identification and characterization of parasitism genes from the pinewood nematode Bursaphelenchus xylophilus reveals a multilayered detoxification strategy. Mol. Plant Pathol. 17, 286-295. doi: 10.1111/mpp. 12280

Fu, Z., Agudelo, P., and Wells, C. E. (2020). Detoxification-related gene expression accompanies anhydrobiosis in the foliar nematode (Aphelenchoides fragariae). J. Nematol. 52, 1-12. doi: 10.21307/jofnem2020-047

Garcia-Brugger, A., Lamotte, O., Vandelle, E., Bourque, S., Lecourieux, D., Poinssot, B., et al. (2006). Early signaling events induced by elicitors of plant defenses. Mol. Plant Microbe Interact. 19, 711-724. doi: 10.1094/MPMI-19-0711

Gillet, F. X., Bournaud, C., Antonino de Souza, J. D. Júnior, and Grossi-de-Sa, M. F. (2017). Plant-parasitic nematodes: towards understanding molecular players in stress responses. Ann. Bot. 119, 775-789. doi: 10.1093/aob/mcw260

Higgins, C. F. (1992). ABC transporters: from microorganisms to man. Annu. Rev. Cell Biol. 8, 67-113. doi: 10.1146/annurev.cb.08.110192. 000435

Islam, S., Rahman, I. A., Islam, T., and Ghosh, A. (2017). Genomewide identification and expression analysis of glutathione S-transferase gene family in tomato: gaining an insight to their physiological and stress-specific roles. PloS ONE 12:e0187504. doi: 10.1371/journal.pone.01 87504

James, C. E., and Davey, M. W. (2009). Increased expression of ABC transport proteins is associated with ivermectin resistance in the model nematode Caenorhabditis elegans. Int. J. Parasitol. 39, 213-220. doi: 10.1016/j.ijpara.2008. 06.009

Jones, J. D., and Dangl, J. L. (2006). The plant immune system. Nature 444, 323-329. doi: 10.1038 /nature 05286

Kadota, Y., Shirasu, K., and Zipfel, C. (2015). Regulation of the NADPH oxidase RBOHD during plant immunity. Plant Cell Physiol. 56, 1472-1480. doi: $10.1093 / \mathrm{pcp} / \mathrm{pcv} 063$

Kikuchi, T., Cotton, J. A., Dalzell, J. J., Hasegawa, K., Kanzaki, N., McVeigh, P., et al. (2011). Genomic insights into the origin of parasitism in the emerging plant pathogen Bursaphelenchus xylophilus. PLoS Pathog. 7:e1002219. doi: 10.1371/journal.ppat.1002219

Kooliyottil, R., Dandurand, L. M., Govindan, B. N., and Knudsen, G. R. (2016). Microscopy method to compare cyst nematode infection of different plant species. Adv. Biosci. Biotechnol. 7, 311-318. doi: 10.4236/abb.2016. 76029

Kooliyottil, R., Dandurand, L. M., Kuhl, J. C., Caplan, A., Xiao, F., Mimee, B., et al. (2019). Transcriptome analysis of Globodera pallida from the susceptible host Solanum tuberosum or the resistant plant Solanum sisymbriifolium. Sci. Rep. 9:13256. doi: 10.1038/s41598-019-49725-6

Kurutas, E. B. (2015). The importance of antioxidants which play the role in cellular response against oxidative/nitrosative stress: current state. Nutr. J. 15:71. doi: 10.1186/s12937-016-0186-5

Laing, R., Bartley, D. J., Morrison, A. A., Rezansoff, A., Martinelli, A., Laing, S. T., et al. (2015). The cytochrome P450 family in the parasitic nematode Haemonchus contortus. Int. J. Parasitol. 45, 243-251. doi: 10.1016/j.ijpara.2014. 12.001

Li, Y., and Meng, F., and Deng, X. (2019). Comparative transcriptome analysis of the pinewood nematode Bursaphelenchus xylophilus reveals the molecular mechanism underlying its defense response to hostderived $\alpha$-pinene. Int. J. Mol. Sci. 20:911. doi: 10.3390/ijms200 40911

Lilley, C. J., Maqbool, A., Wu, D., Yusup, H. B., Jones, L. M., Birch, P. R. J., et al. (2018). Effector gene birth in plant parasitic nematodes: neofunctionalization of a housekeeping glutathione synthetase gene. PLOS Genet. 14:e1007310. doi: 10.1371/journal.pgen.1007310

Lindblom, T. H., and Dodd, A. K. (2009). Errata to: xenobiotic detoxification in the nematode Caenorhabditis elegans. J. Exp. Zool. Part A: Ecol. Genet. Physiol. 311, 312. doi: $10.1002 / \mathrm{jez} .528$

Linton, K. J., and Higgins, C. F. (1998). The Escherichia coli ATPbinding cassette (ABC) proteins. Mol. Microbiol. 28, 5-13. doi: 10.1046/j.1365-2958.1998.00764.x

Lozano, J., and Smant, G. (2011). "Survival of plant-parasitic nematodes inside the host," in Molecular and Physiological Basis of Nematode Survival. eds. R. N. Perry, D. A. Wharton (Oxfordshire: CABI), 28-62. doi: $10.1079 / 9781845936877.0028$

Manosalva, P., Manohar, M., von Reuss, S., Chen, S., Koch, A., Kaplan, F., et al. (2015). Conserved nematode signalling molecules elicit plant defenses and pathogen resistance. Nat. Commun. 6:7795. doi: 10.1038/ncomms8795

Pohl, P. C., Klafke, G. M., and Carvalho, D. D. (2011). ABC transporter efflux pumps: a defense mechanism against ivermectin in Rhipicephalus (Boophilus) microplus. Int. J. Parasitol. 41, 1323-1333. doi: 10.1016/j.ijpara.2011.08.004

Prichard, R. K., and Roulet, A. (2007). ABC transporters and beta-tubulin in macrocyclic lactone resistance: prospects for marker development. Parasitology 134, 1123-1132. doi: 10.1017/S0031182007000091

Qiu, X., Yang, L., Ye, J., Wang, W., Zhao, T., Hu, H., et al. (2019). Silencing of cyp-33C9 gene affects the reproduction and pathogenicity of the pine wood nematode, Bursaphelenchus xylophilus. Int. J. Mol. Sci. 20:4520. doi: $10.3390 /$ ijms 20184520

Robertson, L., Robertson, W. M., Sobczak, M., Helder, J., Tetaud, E., Ariyanayagam, M. R., et al. (2000). Cloning, expression and functional characterisation of a peroxiredoxin from the potato cyst nematode Globodera rostochiensis. Mol. Biochem. Parasitol. 111, 41-49. doi: 10.1016/S0166-6851(00)00295-4

Rosso, L. C. (2009). Cloning, sequence, and expression analysis of a new MnSODencoding gene from the root-knot nematode Meloidogyne incognita. J. Nematol. $41,52-59$.

Sacco, M. A., Koropacka, K., Grenier, E., Jaubert, M. J., and Blanchard, A., Goverse, A., et al. (2009). The cyst nematode SPRYSEC protein RBP-1 elicits Gpa2- and RanGAP2-dependent plant cell death. PLOS Pathog. 5:e1000564. doi: 10.1371/journal.ppat.1000564

Schouteden, N., De Waele, D., Panis, B., and Vos, C. M. (2015). Arbuscular mycorrhizal fungi for the biocontrol of plant-parasitic nematodes: a review of the mechanisms involved. Front. Microbiol. 6:1280. doi: $10.3389 /$ fmicb.2015.01280

Sheps, J. A., Ralph, S., Zhao, Z., Baillie, D. L., and Ling, V. (2004). The ABC transporter gene family of Caenorhabditis elegans has implications for the evolutionary dynamics of multidrug resistance in eukaryotes. Genome Biol. 5:R15. doi: 10.1186/gb-2004-5-3-r15

Shukla, N., Yadav, R., Kaur, P., Rasmussen, S., Goel, S., Agarwal, M., et al. (2018). Transcriptome analysis of root-knot nematode (Meloidogyne incognita)-infected tomato (Solanum lycopersicum) roots reveals complex 
gene expression profiles and metabolic networks of both host and nematode during susceptible and resistance responses. Mol. Plant Pathol. 19, 615-633. doi: $10.1111 / \mathrm{mpp} .12547$

Stitt, L. E., Tompkins, J. B., Dooley, L. A., and Ardelli, B. F. (2011). $\mathrm{ABC}$ transporters influence sensitivity of Brugia malayi to moxidectin and have potential roles in drug resistance. Exp. Parasitol. 129, 137-144. doi: 10.1016/j.exppara.2011.06.018

Xu, M., Molento, M., Blackhall, W., Ribeiro, P., Beech, R., and Prichard, R. (1998). Ivermectin resistance in nematodes may be caused by alteration of P-glycoprotein homolog. Mol. Biochem. Parasitol. 91, 327-335. doi: 10.1016/S0166-6851(97)00215-6
Conflict of Interest: The authors declare that the research was conducted in the absence of any commercial or financial relationships that could be construed as a potential conflict of interest.

Copyright (c) 2020 Kooliyottil, Rao Gadhachanda, Solo and Dandurand. This is an open-access article distributed under the terms of the Creative Commons Attribution License (CC BY). The use, distribution or reproduction in other forums is permitted, provided the original author(s) and the copyright owner(s) are credited and that the original publication in this journal is cited, in accordance with accepted academic practice. No use, distribution or reproduction is permitted which does not comply with these terms. 